\title{
Effects of the Biofeedback Units during PNF Pattern on the Abdominal Muscle Activity and Pelvic Rotation Angle in Patients with Chronic Back Pain
}

\author{
Jin Park \\ Department of Physical Therapy, Drim Sol Hospital, Republic of Korea \\ Received March 31, 2021; Revised May 20, 2021; Accepted June 18, 2021
}

\begin{abstract}
Cite This Paper in the following Citation Styles
(a): [1] Jin Park, "Effects of the Biofeedback Units during PNF Pattern on the Abdominal Muscle Activity and Pelvic Rotation Angle in Patients with Chronic Back Pain," International Journal of Human Movement and Sports Sciences, Vol. 9, No. 5, pp. 847 - 851, 2021. DOI: 10.13189/saj.2021.090503.
\end{abstract}

(b): Jin Park (2021). Effects of the Biofeedback Units during PNF Pattern on the Abdominal Muscle Activity and Pelvic Rotation Angle in Patients with Chronic Back Pain. International Journal of Human Movement and Sports Sciences, 9(5), 847 - 851. DOI: 10.13189/saj.2021.090503.

Copyright $\odot 2021$ by authors, all rights reserved. Authors agree that this article remains permanently open access under the terms of the Creative Commons Attribution License 4.0 International License

\begin{abstract}
Purpose: The purpose of this study was to provide clinical information by comparing abdominal muscle activity and lumbopelvic rotation angle according to proprioceptive neuromuscular facilitation (PNF) lower pattern with pressure biofeedback unit or PNF lower pattern without pressure biofeedback unit in patients with chronic back pain. Methods: In this study, 20 patients with low back pain were instructed to perform PNF lower pattern with biofeedback unit and PNF lower pattern without biofeedback unit. Surface electromyography data were collected from the ipsilateral internal oblique (IIO), contralateral external oblique (CEO), ipsilateral rectus abdominal (IRA) muscles, and lumbopelvic rotation angle were measured using the motion analysis device. Results: The activation of all abdominal muscles was found greater in PNF lower pattern with biofeedback unit than that PNF lower pattern without biofeedback unit. The degree of lumbopelvic rotation angle in PNF lower pattern with biofeedback unit was found lower than that PNF lower pattern without biofeedback unit $(\mathrm{p}<0.05)$. Conclusion: The results of this study suggest that PNF lower pattern with biofeedback unit is effective in increasing abdominal muscle activity and decreasing unwanted lumbopelvic rotation in patients with chronic low back pain. Therefore, if you want to apply the PNF lower pattern to patients with chronic low back pain, it is considered effective to control the pelvis using the biofeedback unit.
\end{abstract}

Keywords Back Pain, Biofeedback Unit, Strength, Surface EMG

\section{Introduction}

Low back pain is a common musculoskeletal disorder, and most people experience low back pain during their lives. Of these, $40 \%$ of people develop chronic low back pain that lasts for at least 12 weeks [1][2]. Such chronic low back pain can be caused by various causes, especially the instability of the lumbar spine and weakening of the lower extremity muscle strength are one of the causes of chronic low back pain [3]. In patients with chronic low back pain, if the lower limbs are repeatedly moved during their daily life, excessive movement in the lumbosacral spine occurs, which soon worsens low back pain. [4][5].

The proprioceptive neuromuscular facilitation (PNF) lower extremity pattern can be applied to strengthen the hip abductor and extensor muscles of the lower extremities. The PNF pattern is an approach that integrates motor control and motor learning. It is an effective method to improve muscle strength, endurance, joint motion range, etc. and to improve daily life movements through pattern movement consisting of diagonal motion [6]. Among the lower limb patterns of the PNF pattern, there are hip 
flexion-abduction-internal rotation patterns, hip extension-adduction-external rotation patterns, and hip extension-abduction-external rotation patterns [7].

However, in the case of chronic low back pain patients, there is a limitation in controlling the erroneous movement of the pelvis during the muscle strengthening exercise of the lower extremities, so an intervention that can improve the stability of the pelvis is needed. In order to improve the stability of the pelvis, it can be classified as a method of using external or internal force. The method of using external force is a method of compressing the sacroiliac joint using the therapist's hand or a pelvic belt, and it can be applied to patients with instability in the lumbar spine to improve stability [8]. However, since the method using external force improves stability only when the therapist's hand or pelvic belt is continuously applied, it is necessary to maintain stability with the patient's internal force [8][9]. The method of using the internal force that can be carried out for this is a method that can control the pelvis by activating the simultaneous contraction of the local muscle and the entire muscle [10]. In a study by Park et al. [11], the subjects were able to directly use the thumb rather than when performing the movement of the lower limb by providing external force through the pelvic belt to the general public. It was reported that the rotation angle of the pelvis decreased during the movement of the lower extremities when the method using the internal force to minimize the movement of the pelvis by palpating anterior superior iliac spine was performed. A study by Noh et al. [12] also suggested that inducing internal force by placing a biofeedback device under the lumbar spine and maintaining pressure during the movement of the lower extremities in patients with chronic low back pain induces the activity of the abdominal muscles and reduces the rotation of the pelvis.

Based on these previous studies, it can be seen that patients with instability of the lumbosacral should reduce the movement of the pelvis through an internal force method because rotation is increased during the lower extremity muscle strength. In patients with chronic low back pain, there may be limitations in selectively mobilizing abdominal muscles or directly controlling pelvic rotation. Therefore, in this study, when applying a PNF lower extremity pattern that can strengthen lower extremity muscle strength, the provision of pressure biofeedback unit activates abdominal muscle activity and attempts to provide clinical information by verifying the effect on the change of pelvic rotation angle.

\section{Methods}

\subsection{Subjects}

A total of 20 patients who were admitted to the rehabilitation hospital located in $\mathrm{J}$ city, Korea, who understood the purpose of the study and wrote a consent form to participate in the study based on the declaration of Helsinki, were conducted. The inclusion criteria of the study were those who have not had a history of back surgery, and those whose visual analogue scale (VAS) lasts more than 3 points due to pain in the lower back for more than 3 months and low back pain. This study was performed on patients who had an oswestry disability index (ODI) of 7 or higher and were diagnosed with chronic back pain by a specialist. Table 1 shows the general characteristics of the subjects.

Table 1. General characteristics of subjects $(n=20)$

\begin{tabular}{cc}
\hline Characteristics & Back pain group \\
\hline Age (years) & $44.53 \pm 9.76^{\mathrm{a}}$ \\
Gender (M/F) & $8 / 12$ \\
Height (cm) & $166.11 \pm 8.94$ \\
Weight (kg) & $64.27 \pm 9.87$ \\
BMI (score) & $23.47 \pm 3.54$ \\
\hline
\end{tabular}

${ }^{\mathrm{a}}$ Mean \pm standard deviation

\subsection{Procedure}

A pressure biofeedback unit (Stabilizer, Chattanooga Group Inc, USA) was used to provide trunk stabilization feedback when applying the PNF lower extremity pattern. Subjects placed the pressure biofeedback unit between the floor and the waist in the side lying position and held the scale with one hand to check the pressure to $40 \mathrm{mmHg}$, and the pressure was maintained between $35 \mathrm{mmHg}$ and $45 \mathrm{mmHg}$ while performing the PNF pattern. So that the trunk can be stably maintained [13]. PNF lower limb pattern application according to the provision of pressure biofeedback and PNF lower limb pattern application in the state of not providing pressure biofeedback, respectively, were performed to measure abdominal muscle activity through EMG and pelvic rotation angle in cross section. Resting time between each condition was 5 minutes, and muscle fatigue of the subjects was prevented.

Subjects were placed on the treatment bed with the dominant side up, and the torso, pelvis, and legs were aligned. In order to effectively strengthen the hip abductor and hip extensors, an extension-abduction-internal rotation pattern of the dominant lower limb was applied, and the last posture was kept for 5 seconds. The application of the PNF pattern was conducted by a researcher who completed level $3 \mathrm{a}$ of education conducted by the Society for PNF.

\subsection{Assessment}

\subsubsection{Muscle Activity}

Application of the PNF lower limb pattern to the subjects' dominant feet to measure the muscle activity of 
the ipsilateral internal oblique abdominal muscle, the opposite external oblique abdominal muscle, and the ipsilateral rectus abdominis muscle in the last posture, a surface electromyography device (TeleMyo DTS, Noraxon EMG, USA) was used. To remove noise that may occur during the EMG measurement, the electrode attachment area was shaved and sterilized with an alcohol cotton pad. Surface electrodes (Ag-AgCl, Bioprotech, South Korea) were attached to each muscle area at $1.5 \mathrm{~cm}$ intervals according to the surface EMG guidelines. In order to obtain the reference values of the internal abdominal, external abdominal, and rectus abdominal muscles, the average value was measured by raising both feet of about $8 \mathrm{~cm}$ from the table for 3 seconds in a straight position with the knee bent, and the reference voluntary contraction (\%RVC) of muscle contraction during motion was obtained [14].

For EMG measurements, a band-pass filter of $20-450 \mathrm{~Hz}$ and a notch filter of $60 \mathrm{~Hz}$ were used [15]. In addition, a sampling rate of $1,000 \mathrm{~Hz}$ was applied. The EMG measurements were made using dedicated software (MyoResearch XP Master 1.06 software, Noraxon, USA), and the measured EMG signals were converted into root mean square (RMS). The average value was statistically processed by measuring three times.

\subsubsection{Pelvic Rotation}

A motion analysis device (Motion biofeedback device, Relive, Korea) was used to measure the change in the pelvic rotation angle in the cross section of the subjects during the PNF lower limb pattern. The motion analysis device was fixed with a band between the anterior superior iliac spine and the anterior inferior iliac spine on the opposite side of the subject. Changes in the angle of rotation of the pelvis during the PNF lower extremity pattern were measured on subjects [16]. The mean value was statistically processed by measuring three times.

\subsection{Statistical Analysis}

All statistical analyzes of this study were conducted using the SPSS software (ver 22.0. SPSS Inc, Chicago, USA). An independent t-test was performed to analyze changes in muscle activity of each muscle and pelvic rotation angle in the cross section according to the presence or absence of pressure biofeedback during the PNF lower limb pattern. All statistical significance levels (p) of the data were tested as 0.05 .

\section{Results}

\subsection{Abdominal Muscle Activity}

It was found that the muscle activity of the internal abdominal, external abdominal, and rectus abdominal muscle was statistically significantly improved when the
PNF pattern was performed with the biofeedback unit compared to the PNF pattern without the biofeedback unit $(\mathrm{p}<0.05)($ Table 2).

Table 2. EMG activity (\%RVC) of abdominal muscles

\begin{tabular}{cccc}
\hline Muscles & $\begin{array}{c}\text { With } \\
\text { biofeedback unit }\end{array}$ & $\begin{array}{c}\text { Without } \\
\text { biofeedback unit }\end{array}$ & $\mathrm{p}$ \\
IIO $^{\mathrm{a}}$ & $60.26 \pm 23.40^{\mathrm{d}}$ & $33.77 \pm 18.79$ & $0.01^{*}$ \\
$\mathrm{CEO}^{\mathrm{b}}$ & $133.01 \pm 58.81$ & $81.67 \pm 47.45$ & $0.04^{*}$ \\
IRA $^{\mathrm{c}}$ & $99.11 \pm 16.13$ & $71.46 \pm 16.05$ & $0.01^{*}$ \\
\hline
\end{tabular}

${ }^{\mathrm{a}}$ Ipsilateral internal oblique, ${ }^{\mathrm{b}}$ Contralateral external oblique, ${ }^{\mathrm{C}}$ Ipsilateral rectus abdominal, ${ }^{\mathrm{d}}$ Mean \pm standard deviation, significant difference between the change values among the group $\left({ }^{*} \mathrm{p}<0.05\right)$

\subsection{Pelvic Rotation Angle}

The pelvic rotation angle was found to be statistically significantly reduced when the PNF pattern was performed with the biofeedback unit provided compared to when the PNF pattern was performed without the biofeedback unit $(\mathrm{p}<0.05)($ Table 3$)$.

Table 3. The angle of pelvic rotation

\begin{tabular}{cccc}
\hline & $\begin{array}{c}\text { With } \\
\text { biofeedback unit }\end{array}$ & $\begin{array}{c}\text { Without } \\
\text { biofeedback unit }\end{array}$ & $\mathrm{p}$ \\
\hline $\begin{array}{c}\text { Pelvic } \\
\text { rotation }\end{array}$ & $8.25 \pm 1.60^{\mathrm{a}}$ & $11.30 \pm 2.73$ & $0.00^{*}$ \\
\hline
\end{tabular}

${ }^{a}$ Mean \pm standard deviation, significant difference between the change values among the group $\left({ }^{*} \mathrm{p}<0.05\right)$

\section{Discussion}

This study investigated the effect of using a pressure biofeedback unit to control trunk stability on the change of abdominal muscle activity and pelvic rotation angle when applying the PNF pattern to strengthen the hip abductor muscle and hip extensor muscle in chronic low back pain patients. This study was conducted to provide a clinical information for rehabilitation of patients with chronic low back pain.

As a result, it was found that providing pressure biofeedback unit during the PNF lower extremity pattern in all of the internal oblique abdominal, external oblique abdominal, and rectus abdominal muscles showed higher muscle activity compared to the state not provided. The trunk plays a central role in the functional activity chain and is very important as it is the basis for limb movement [17]. In particular, the stabilization of the trunk shows an increase in the stability of the lower extremity joint and an improvement in muscle strength due to increased muscle fiber mobilization [18]. In a study by Cynn et al.[19], it was found that performing hip abduction exercise after stabilizing the trunk by providing pressure biofeedback in the supine position was effective in improving the abdominal muscle activity. This is because the activity of 
the abdominal muscles is increased to ensure the stability of the trunk while moving the lower limbs. In a study by Yoo et al.[20], it was found that the application of the PNF lower limb pattern in the state of applying the abdominal hollowing increases the muscle activity of the abdominal muscles and lower extremities than when the abdominal hollowing is not applied. This is a result of the increase in the activity of the abdominal muscles during the movement of the lower extremities. Internal and external forces are required for the stability of the lumbar spine during the movement of the lower extremities. In the study of $\mathrm{Hu}$ et al.[9], the stability of the lumbosacral spine is secured by the internal force caused by contraction of the transverse abdominal and oblique muscles during the lower limb movement. In this study, it is also thought that the internal force and the stability of the lumbar spine were increased by the activation of the abdominal muscles according to the provision of pressure biofeedback.

As a result of measuring the change in pelvic rotation angle, it was found that providing a biofeedback unit during the PNF lower extremity pattern was effective in reducing the pelvic rotation angle compared to that without providing a biofeedback unit. The reduction of rotation of the pelvis during the movement of the lower extremities is an important consideration for patients with chronic low back pain, which means that securing the stability of the trunk using a biofeedback unit is effective in reducing the rotation angle. A study by Noh et al.[12] also reported that the pelvic rotation angle decreased when an internal force was induced using the biofeedback unit during the movement of the lower extremities in women with chronic low back pain. It was reported that the internal force caused by the lumbosacral spine was involved in the stability of the lumbar vertebrae and reduced rotation. In order to decrease the pelvic rotation angle during the movement of the lower extremities, proper activity of the abdominal muscles is required. In particular, the pelvic rotation angle decreases due to the activation of the ipsilateral internal oblique abdominal and opposite external oblique muscles [11][21]. Based on the previous studies and the results of this study, it is thought that during the PNF lower extremity pattern, the biofeedback unit was used to elicit muscle activity of the abdominal muscles, thereby increasing the stability of the lumbosacral spine, resulting in decreased pelvic rotation.

The subjects of this study were able to apply the pattern directly because the muscle strength of the abductor and extensor of the hip joint on the dominant side was more than a certain standard. However, in the case of chronic low back pain patients with severe pain or weak muscle strength, there may be limitations in directly applying the pattern. In addition, it is necessary to verify this as evaluation is only conducted after the intervention, and there is no evaluation on whether the effect continues.

This study was conducted to verify the effect on abdominal muscle activity and pelvic rotation angle by using a biofeedback unit to control trunk stability during PNF lower extremity pattern for strengthening lower extremity muscle strength in chronic low back pain patients. Using a biofeedback unit to secure the stability of the trunk and performing the PNF lower limb pattern was shown to be an effective method for reducing abdominal muscle activity and pelvic rotation angle. Therefore, the use of a biofeedback unit should be considered in order to secure the stability of the trunk while performing the PNF lower limb pattern or movement of the lower limb in clinical practice.

\section{Conflicts of Interest}

The authors declare no conflicts of interest.

\section{REFERENCES}

[1] Airaksinen O, Brox JI, Cedraschi C, et al. European guidelines for the management of chronic nonspecific low back pain. European Spine Journal. 2006;15(Suppl2):s192

[2] Costa LDCM, Maher CG, McAuley JH, et al. Prognosis for patients with chronic low back pain: inception cohort study. British Medical Journal. 2009;339:b3829

[3] Panjabi MM. Clinical spinal instability and low back pain. Journal of Electromyography and Kinesiology. 2003;13(4):371-379

[4] Hoffman SL, Johnson MB, Zou D, Harris-Hayes M, Van Dillen R. Effect of classification-specific treatment on lumbopelvic motion during hip rotation in people with low back pain. Manual Therapy. 2011;16(4):344-350

[5] Roussel NA, Nijs J, Mottram S, Van Moorsel A, Truijen S, Stassijns G. Altered lumbopelvic movement control but not generalized hypermobility is associated with increased injury in dancers. A prospective study. Manual Therapy. 2009;14(6):630-635

[6] Kofotolis ND, Kellis E. Cross-training effects of a proprioceptive neuromuscular facilitation exercise programme on knee musculature. Physical Therapy in Sport. 2007;8(3):109-116

[7] Alder SS, Beckers D, Buck M. PNF in practice: an illustrated guide, 4th ed. Berlin. Springer-Verlag. 2014

[8] Takasaki H, Iizawa $\mathrm{T}$, Hall $\mathrm{T}$, et al. The influence of increasing sacroiliac joint force closure on the hip and lumbar spine extensor muscle firing pattern. Manual Therapy. 2009;14(5):484-489

[9] Hu H, Meijer OG, van Dieen JH, et al. Muscle activity during the active straight leg raise (ASLR), and the effects of a pelvic belt on the ASLR and on treadmill walking. Journal of Biomechanics. 2010;43(3):532-539

[10] Richardson CA, Snijders CJ, Hides JA, et al. The relation between the transversus abdominis muscles, sacroiliac joint mechanics, and low back pain. Spine. 2002;27(4):399-405 
[11] Park KH, Ha SM, Kim SJ, et al. Effects of the pelvic rotatory control method on abdominal muscle activity and the pelvic rotation during active straight leg raising. Manual Therapy. 2013;18(3):220-224

[12] Noh KH, Kim JW, Kim GM, et al. The influence of dual pressure biofeedback units on pelvic rotation and abdominal muscle activity during the active straight leg raise in women with chronic lower back pain. Journal of Physical Therapy Science. 2014;26(5): 717-719

[13] McBeth JM, Earl-Boehm JE, Cobb SC, et al. Hip muscle activity during 3 side-lying hip-strengthening exercises in distance runners. Journal of Athletic Training. 2012;47(1):15-23

[14] Dankaerts W, O'sullivan PB, Burnett AF, et al. Reliability of EMG measurements for trunk muscles during maximal and sub-maximal voluntary isometric contractions in healthy controls and CLBP patients. Journal of Electromyography Kinesiology. 2004;14(3): 333-342

[15] Winter DA. Biomechanics and motor control of human movement. Hoboken, john Wiley \& Sons, Inc. 2009

[16] Liebenson C, Karpowicz AM, Brown SH, et al. The active straight leg raise test and lumbar spine stability. Archives of Physical Medicine and Rehabilitation. 2009;1(6):530-535
[17] Jacob K. Gardner , Justin T. Chia , Kelsey L. Miller , "Leg-drive Does Not Affect Upper Extremity Muscle Activation during a Bench Press Exercise," International Journal of Human Movement and Sports Sciences, Vol. 7, No. 1, pp. 12 - 17, 2019. DOI: 10.13189/saj.2019.070103

[18] Akuthota V, Nadler SF. Core strengthening. Archives of Physical Medicine and Rehabilitation. 2004;85(3 Suppl 1):86-92

[19] Cynn HS, Oh JS, Kwon OY, et al. Effects of lumbar stabilization using a pressure biofeedback unit on muscle activity and lateral pelvic tilt during hip abduction in sidelying. Archives of Physical Medicine and Rehabilitation. 2006;87(11):1454-1458

[20] Yoo B, Park H, Heo K, et al. The effects of abdominal hollowing in lower limb PNF pattern training on the activation of contralateral muscles. Journal of Physical Therapy Science. 2013;25(10): 1335

[21] Nelson-Wong E, Poupore K, Ingvalson S, et al. Neuromuscular strategies for lumbopelvic control during frontal and sagittal plane movement challenges differ between people with and without low back pain. Journal of Electromyography and Kinesiology. 2013;23(6):1317-1324 